\title{
A Comparative Study of Calcified Epithelioma of Malherbe And Epidermoid Cyst With Ultrasound
}

Xiuliang Wei

Shandong University

Dongfeng Cheng

Jinan Fourth People's Hospital

Chunchun Shao

Shandong University

Kunkun Pang

Shandong University

Juan Xiao

Shandong University

Yuqing Zhang

Shandong University

Mei Wu

Shandong University

Lulu Zhang

Shandong University

Pingjuan Ni

Shandong University

Yu Liang

Shandong University

Feixue Zhang ( $\nabla$ snow-1860@163.com )

Shandong University

\section{Research Article}

Keywords: Calcifying epithelioma of Malherbe, epidermoid cyst, subcutaneous tissues, ultrasound, ultrasonic features

Posted Date: January 31st, 2022

DOI: https://doi.org/10.21203/rs.3.rs-1274068/v1

License: (9) This work is licensed under a Creative Commons Attribution 4.0 International License. Read Full License 
Page $2 / 20$ 


\section{Abstract}

Objective: To explore the ultrasonic(US)features of calcified epithelioma Malherbe (CEM) and epidermoid cyst(EC) in differential diagnosis and improve the accuracy of US diagnosis of CEM.

Materials and Methods: We retrospectively analyzed 309 subjects, who underwent US examination before operation and pathological diagnosis for CEM or EC post-operation. The subjects were categorized into the training and validation sets according to different inspection times. The US and clinical features of CEM were evaluated through univariate analysis. A multivariate logistic regression model was used to establish the diagnostic model.

Results: Statistically significant variables in univariate analysis $(P<0.05)$ were included in the multivariate logistic regression model. The results demonstrated that the diagnostic model for CEM was statistically significant $(P<0.001)$. The risk factors included posterior echo attenuation and hypoechoic halos $(\mathrm{OR}=9.277,10.254)$ and the protective factors included age, thickness diameter, and posterior echo enhancement $(\mathrm{OR}=0.936,0.302,0.156)$. The diagnostic models tested in the training set (AUC=0.974, 95\% $\mathrm{Cl}=0.955-0.994)$ and the validation $\operatorname{set}(\mathrm{AUC}=0.967,95 \% \mathrm{Cl}=0.926-1.000)$ demonstrated good discriminant ability.

Conclusions: The diagnostic accuracy for CEM is higher compared to EC when the nodule is characterized by posterior echo attenuation, hypoechoic halos, smaller thickness, and younger age. US diagnostic model can guide the diagnosis of CEM.

\section{Introduction}

Calcifying epithelioma of Malherbe (CEM), also known as pilomatrixoma (PM), is a benign tumor that stems from hair stromal cells, mostly found between the dermis and the subcutaneous fat layer ${ }^{1,2}$. In most cases, CEM grows gradually but may also grow rapidly with local aggression. The incidence of CEM is low, about 1/800-1000; also, the coincidence rate of ultrasonic diagnosis is low ${ }^{3,4}$. An epidermoid cyst $(E C)$ is among the most common tumors that grow in the skin or superficial subcutaneous tissue. Being a cystic lesion, EC is easily misdiagnosed owing to the variation and complexity of the ultrasonic manifestations ${ }^{5-7}$. Of note, CEM and EC are both derived from epidermal tissue; however, CEM requires extensive excision to ensure a thorough radical cure, whereas EC requires that the whole lesion be removed ${ }^{1,8,9}$. Therefore, accurate diagnosis of the two diseases is highly imperative for the choice of correct surgical methods. Reports have demonstrated the high accuracy of high-frequency ultrasound in the diagnosis of superficial lesions ${ }^{1-3}$. The authors found that the misdiagnosis rate of CEM was high in clinical practice. By analyzing ultrasound diagnostic results of the cases, which the pathological findings were CEM over the past 5 years, we found that the correct rate of diagnosis was $38.2 \%$ with ultrasound, whereas the misdiagnosis rate was $61.8 \%$ (EC $52.4 \%$ and other lesions $47.6 \%$ ). Nearly half of CEM cases were misdiagnosed as EC. Despite suggestions from previous studies that intramural calcification is a crucial ultrasonic feature of CEM, the actual role it plays in the differentiation of CEM from EC is unclear. 
Moreover, the contribution of the characteristic changes of EC, such as onionskin sign, fissure sign, apple sign, dotted and striped hyperecho to the differentiation is elusive ${ }^{8,10-12}$. Some scholars found that the length, width, and thickness of EC were larger than CEM 13,14; however, the value of these three diameters in the differential diagnosis is yet to be explored. Herein, we aimed to construct a diagnostic model of CEM through a comparison of the clinical and ultrasonic characteristics between CEM and EC and improve the accuracy of ultrasonic diagnosis of CEM.

\section{Results}

\section{Basic demographic characteristics of the training set and validation set}

To construct the diagnostic model, we enrolled 206 cases, including 49 cases of CEM and 157 cases of EC in the training set (age range, 0-78 years) between December 2015 and March 2019. Besides, 103 cases, including 19 cases of CEM and 84 cases of EC in the validation set (age range, 0.5-76 years) between April 2019 and December 2020, were applied to validate the performance of the diagnostic model. Findings suggested no significant differences between the two groups in terms of age, sex, and pathology results ( $P>0.05$, Table 1$)$, except for the onset time, which was statistically significant $(P<0.05$, Table 1).

Table 1

Demographic characteristics of training set and validation set

\begin{tabular}{|lllll|}
\hline Factors & $\begin{array}{l}\text { Training set } \\
(\mathbf{n}=206)\end{array}$ & Validation set(n=103) & $W / \chi^{2}$ & $P$ \\
\hline Age & $34.50(15.25,52.75)$ & $40.00(27.00,54.50)$ & 9528 & 0.144 \\
\hline Onset time & $24.00(5.00,54.00)$ & $36.00(12.00,93.00)$ & 4324 & $\mathbf{0 . 0 0 2}$ \\
\hline Sex & & & & \\
\hline Male & $128(62.4 \%)$ & $74(71.8 \%)$ & 2.687 & 0.101 \\
\hline Female & $77(37.6 \%)$ & $29(28.2 \%)$ & & \\
\hline Pathology results & & & & \\
\hline EC & $157(76.2 \%)$ & $84(81.5 \%)$ & 1.141 & 0.285 \\
\hline CEM & $49(23.8 \%)$ & $19(18.5 \%)$ & & \\
\hline
\end{tabular}

\section{Clinical characteristics of the training set}


We explored the clinical characteristics of CEM and EC in the training set and found that the differences in age, onset time, and location were statistically significant $(P<0.05$, Table 2$)$. However, differences in gender, skin color, skin damage, and skin adhesion were not statistically significant ( $P \bowtie 0.05$, Table 2$)$. 
Table 2

Univariate analysis results of clinical features of CEM and EC in the training set

\begin{tabular}{|lllll|}
\hline Factors & $\begin{array}{l}\text { CEM } \\
(\mathbf{n}=49)\end{array}$ & $\begin{array}{l}\text { EC } \\
(\mathbf{n}=157)\end{array}$ & W/ $\chi^{2}$ & $P$ \\
\hline Age & $9.00(6.00,15.00)$ & $43.00(29.00,56.00)$ & 1062.5 & $<0.001$ \\
\hline Sex & & & & \\
\hline Male & $27(55.1 \%)$ & $101(64.7 \%)$ & 1.478 & 0.224 \\
\hline Female & $22(44.9 \%)$ & $55(35.3 \%)$ & & \\
\hline Onset time(m) & $5.50(2.00,12.00)$ & $24.00(6.00,60.00)$ & 958.5 & 0.005 \\
\hline Skin color & & & & \\
\hline Normal & $38(77.6 \%)$ & $121(77.1 \%)$ & 2.72 & 0.843 \\
\hline Red & $7(14.3 \%)$ & $28(17.8 \%)$ & & \\
\hline Blue & $2(4.1 \%)$ & $2(1.3 \%)$ & & \\
\hline Gray black & $1(2.0 \%)$ & $2(1.3 \%)$ & & \\
\hline Dark red & $0(0.0 \%)$ & $1(0.6 \%)$ & & \\
\hline Buergers & $1(2.0 \%)$ & $2(1.3 \%)$ & & \\
\hline Blackhead & $0(0.0 \%)$ & $1(0.6 \%)$ & & \\
\hline Skin damage & & & & \\
\hline Without & $43(87.8 \%)$ & $141(89.8 \%)$ & 0.779 \\
\hline With & $6(12.2 \%)$ & $16(10.2 \%)$ & & \\
\hline Skin adhesion & & & & \\
\hline Without & $42(85.7 \%)$ & $137(87.3 \%)$ & & \\
\hline With & $7(14.3 \%)$ & $20(12.7 \%)$ & & \\
\hline Location & & & & \\
\hline Head and face & $8(16.3 \%)$ & $26(16.6 \%)$ & & \\
\hline Neck & $19(38.8 \%)$ & $25(15.9 \%)$ & & \\
\hline Trunk & $3(6.1 \%)$ & $36(22.9 \%)$ & & \\
\hline Limbs & $17(34.7 \%)$ & $28(17.8 \%)$ & & \\
\hline Buttocks & $2(4.1 \%)$ & $30(19.2 \%)$ & & \\
\hline Axillary fossa & $0(0.0 \%)$ & $6(3.8 \%)$ & & \\
\hline
\end{tabular}




\begin{tabular}{|llll|}
\hline Factors & $\begin{array}{l}\text { CEM } \\
(\mathrm{n}=49)\end{array}$ & $\begin{array}{l}\text { EC } \\
(\mathrm{n}=157)\end{array}$ & $W / x^{2} \quad P$ \\
\hline Groin & $0(0.0 \%)$ & $6(3.8 \%)$ & \\
\hline
\end{tabular}

\section{Ultrasonic characteristics of the training set}

The length $(L)$, width, and thickness $(T)$ of CEM were: range, $0.5-5.3 \mathrm{~cm}, 0.4-4.3 \mathrm{~cm}$, and $0.3-3.0 \mathrm{~cm}$; median, $1.3 \mathrm{~cm}, 1.0 \mathrm{~cm}, 0.7 \mathrm{~cm}$, respectively. The length, width and thickness of EC were: range, $0.4-22.3 \mathrm{~cm}, 0.3-$ $20.0 \mathrm{~cm}$ and $0.2-16.2 \mathrm{~cm}$; median, $2.2 \mathrm{~cm}, 1.8 \mathrm{~cm}, 1.0 \mathrm{~cm}$, respectively. Except for $\mathrm{L} / \mathrm{T}$, echo, the other variables exhibited not statistically significant differences between the two groups $(P<0.05$, Table 3$)$. 
Table 3

Univariate analysis results of ultrasonic characteristics of CEM and EC in the training set

\begin{tabular}{|c|c|c|c|c|}
\hline Factors & $C E(n=49)$ & $E C(n=157)$ & $w / x^{2}$ & $P$ \\
\hline Length $(\mathrm{cm})$ & $1.30(1.00,1.60)$ & $2.20(1.30,3.50)$ & 2143 & $<0.001$ \\
\hline Width (cm) & $1.00(0.80,1.20)$ & $1.80(1.10,2.70)$ & 2034.5 & $<0.001$ \\
\hline Thickness(cm) & $0.70(0.50,0.80)$ & $1.00(0.70,1.60)$ & 2043.5 & $<0.001$ \\
\hline $\mathrm{L} / \mathrm{T}$ & $1.80(1.50,2.50)$ & $1.93(1.59,2.33)$ & 3573 & 0.453 \\
\hline \multicolumn{5}{|l|}{ Echogenicity } \\
\hline Hypoechoic & $33(67.4 \%)$ & $78(49.7 \%)$ & 8.856 & 0.065 \\
\hline Hyperechoic & $4(8.2 \%)$ & $7(4.5 \%)$ & & \\
\hline Isoechoic & $1(2.0 \%)$ & $3(1.9 \%)$ & & \\
\hline Anechoic & $8(16.3 \%)$ & $61(38.8 \%)$ & & \\
\hline Mixed & $3(6.1 \%)$ & $8(5.1 \%)$ & & \\
\hline \multicolumn{5}{|c|}{ Parenchyma echo } \\
\hline Homogeneous & $3(6.1 \%)$ & $41(26.1 \%)$ & 8.886 & 0.003 \\
\hline Heterogeneous & $46(93.9 \%)$ & $116(73.9 \%)$ & & \\
\hline \multicolumn{5}{|l|}{ Morphology } \\
\hline Round or oval & $42(85.7 \%)$ & $151(96.2 \%)$ & 6.916 & 0.009 \\
\hline Irregular & $7(14.3 \%)$ & $6(3.8 \%)$ & & \\
\hline \multicolumn{5}{|l|}{ Boundary } \\
\hline Distinct & $35(71.4 \%)$ & $150(95.5 \%)$ & & $<0.001$ \\
\hline Indistinct & $14(28.6 \%)$ & $7(4.5 \%)$ & & \\
\hline \multicolumn{5}{|l|}{ Posterior echo } \\
\hline Enhanced & $7(14.3 \%)$ & $125(79.6 \%)$ & 97.505 & $<0.001$ \\
\hline Attenuated & $29(59.2 \%)$ & $5(3.2 \%)$ & & \\
\hline Invariant & $13(26.5 \%)$ & $27(17.2 \%)$ & & \\
\hline \multicolumn{5}{|l|}{ Calcification } \\
\hline Without & $11(22.5 \%)$ & $134(85.4 \%)$ & 82.565 & $<0.001$ \\
\hline Dotted & $28(57.1 \%)$ & $13(8.3 \%)$ & & \\
\hline
\end{tabular}




\begin{tabular}{|lllll|}
\hline Factors & CE(n=49) & EC(n=157) & $W / \chi^{2}$ & $P$ \\
\hline Patchy & $5(10.2 \%)$ & $10(6.4 \%)$ & & \\
\hline Full & $5(10.2 \%)$ & $0(0.0 \%)$ & & \\
CDFI & & & & \\
\hline Without & $25(51.0 \%)$ & $119(75.8 \%)$ & 16.942 & 0.001 \\
\hline Peripheral & $4(8.2 \%)$ & $15(9.6 \%)$ & & \\
\hline Internal & $15(30.6 \%)$ & $14(8.9 \%)$ & & \\
\hline Both of them & $5(10.2 \%)$ & $9(5.7 \%)$ & & \\
\hline Special sign & & & & \\
\hline Fissure sign & $2(4.1 \%)$ & $25(15.9 \%)$ & 13.647 & 0.009 \\
\hline Onionskin sign & $0(0.0 \%)$ & $7(4.5 \%)$ & & \\
\hline Apple sign & $0(0.0 \%)$ & $3(1.9 \%)$ & & \\
\hline Dotted and strip hyperechogenicity & $8(16.3 \%)$ & $41(26.1 \%)$ & & \\
\hline Without & $39(79.6 \%)$ & $81(51.6 \%)$ & & \\
\hline Hypoechoic halo sign & & & & \\
\hline Without & $17(34.7 \%)$ & $137(87.1 \%)$ & 54.685 & $<.001$ \\
\hline With & $32(65.3 \%)$ & $20(12.9 \%)$ & & \\
\hline
\end{tabular}

\section{Multivariate logistic regression analysis of the training set}

The variables with statistical significance in univariate analysis $(P<0.05)$ were included in the multivariate logistic regression model as independent variables, the diagnostic model demonstrated statistical significance $\left(\chi^{2}=142.956, P \otimes 0.001, R^{2}=0.746\right)$. Posterior echo attenuation and hypoechoic halos sign were risk factors for diagnosis of CEM, whereas age, thickness diameter, and posterior echo enhancement were protective factors for diagnosis of CEM (OR=9.277, 10.254, 0.936, 0.302, 0.156, $P<0.05$, Table 4). 
Table 4

Multivariate logistic regression model of CEM and EC

\begin{tabular}{|c|c|c|c|}
\hline Variable & $\beta$ & $\mathrm{OR}(95 \% \mathrm{Cl})$ & $P$ \\
\hline Age & -0.066 & $0.936(0.903,0.971)$ & $凶 0.001$ \\
\hline Thickness & -1.198 & $0.302(0.009,0.917)$ & 0.035 \\
\hline \multicolumn{4}{|c|}{ Posterior echo } \\
\hline Invariant & Reference & Reference & \\
\hline Enhanced & -1.856 & $0.156(0.043,0.574)$ & 0.004 \\
\hline Attenuated & 2.228 & $9.277(1.896,45.396)$ & 0.019 \\
\hline \multicolumn{4}{|c|}{ Hypoechoic halo sign } \\
\hline Without & Reference & Reference & \\
\hline With & 2.328 & $10.254(2.979,35.295)$ & $凶 0.001$ \\
\hline
\end{tabular}

\section{Nomogram of ultrasound diagnostic model for CEM}

A nomogram of the ultrasound diagnostic model for CEM was constructed according to the multivariate analysis of the training set (Figure 2). Then, we calculated the sum of the variable points through the nomogram to estimate the probability to diagnose CEM (Figure 3-4).

\section{ROC curve of the training and validation sets}

Through the calculation of the maximum Youden index and the ROC curve analysis of the training set, we confirmed 0.632 as the cut-off value of the diagnostic model, with $94.3 \%$ sensitivity and $91.8 \%$ specificity. The AUC of the training set was 0.974 (95\% Cl: 0.955-0.994), (Figure 5). The diagnostic model demonstrated $92.9 \%$ sensitivity and $89.5 \%$ specificity in the validation set. The AUC of the validation set was 0.967 (95\% Cl: 0.926-1.000) (Figure 6).

\section{Discussion}

The present study has revealed the risk factors for the diagnostic model of CEM, including posterior echo attenuation ( $O R=9.277)$ and nodular hypoechoic halos $(O R=10.254)$, and the protective factors, including age $(\mathrm{OR}=0.936)$, thickness $(\mathrm{OR}=0.302)$, and posterior echo enhancement $(\mathrm{OR}=0.156)$. Considering the ultrasonic and clinic features, our diagnostic model demonstrated the presence of CEM at the cut-off point of 0.632 with $94.3 \%$ sensitivity and $91.8 \%$ specificity for the training set and $92.9 \%$ sensitivity and $89.5 \%$ specificity for the validation set at the cut-off point of 0.460 . The findings revealed that the ultrasound diagnostic model for CEM can help clinicians distinguish CEM from EC. 
Although CEM and EC can occur at any age, this work revealed that CEM was more common in children and adolescents, whereas EC was prevalent in young and middle-aged adults. The median age of onset was 9 years and 43 years. The median time from discovery to the removal of the lesion was 5.5 months and 24 months for CEM and EC, respectively. The course of CEM disease was much less compared to that of EC, which is potentially related to the younger age of onset of CEM and much attention from family members. We also revealed that age is a protective factor, that is, older patients are less likely to be diagnosed with CEM, which is consistent with previous findings ${ }^{15,16}$.

Furthermore, this study demonstrated that the length, width, thickness of the nodules of CEM were smaller than EC $(P<0.001)$, which concur with previous studies ${ }^{13,14}$. The multivariate logistic regression model implicated thickness as a risk factor for CEM diagnosis. As the thickness reduce, it is more likely to diagnose CEM. However, there are no existing literature reports on whether the thickness is more valuable for the diagnosis of CEM.

Both CEM and EC belong to subcutaneous and superficial masses but their pathological basis is different. Notably, ultrasonic characteristics are closely related to the pathological basis. Pathologically, CEM comprises mainly the basophil cells and shadow cells. The composition ratio of the two varies in different stages. For instance, the shadow cells increase with time, which induces characteristic calcification and osteogenesis ${ }^{17-21}$. In most cases, the pathological manifestations of EC are substantial lesions with increased keratides and squamous changes in lining keratides ${ }^{22,23}$. Herein, we found that $64.4 \%$ CEM was characterized by spotty and/or patchy strong echo, which was consistent with the pathological findings. Previous studies had reported that calcification inside the nodule, including, internal strong echo, was a crucial ultrasonic feature of CEM ${ }^{24,25}$. However, we did not include this feature in the diagnostic model because it was less predictive than other ultrasonic features in the AUC analysis. Calcification does not play a key role in the differential diagnosis between CEM and EC. The finding was different from previous evidence.

In the present study, $55.6 \%$ of the nodules exhibited reduced posterior echo. Some experts believe that posterior echo attenuation is potentially associated with calcification within the nodules or the increase of shadow cells within the non-calcified nodules ${ }^{4,19}$. EC is referred to as cysts, though their ultrasound appearances are not typical of anechoic nodules and may differ based on the number of keratides ${ }^{15,26}$. We could observe hyperechoic, hypoechoic, isoechoic, mixed echoic, and anechoic nodules, however, $78.7 \%$ of EC showed posterior echo enhancement. This could be related to the low attenuation of the acoustic beam passing through the EC. Previously, it was revealed that the degree of enhancement was associated with the water content in the cyst and the distribution of keratinic substances ${ }^{27}$. Our study has implicated posterior echo attenuation as a risk factor for CEM, whereas posterior echo enhancement is a protective factor. These findings concur with their pathological basis and are among the important characteristics of CEM and EC differentiation. Compared to calcification, posterior echo attenuation is more important in the diagnosis of CEM. 
In addition, we revealed that the hypoechoic halo is an important factor in the diagnosis of CEM, and it is closely related to its pathological basis. Choo et al. found that the hypoechoic halo represented the connective tissue sac around the nodules ${ }^{1,28}$. Another study by Solivetti et al. revealed that this was attributed to the calcium deposition in CEM, which triggered the surrounding inflammatory response. Consequently, these events induced the proliferation of small blood vessels in the surrounding tissues and reduced the echo adjacent to the nodules ${ }^{4}$. Elsewhere, DeRosa et al. suggested that CEM is surrounded by arterioles, a finding that concurs with the views of Solivetti ${ }^{4,29}$. In the present study, $66.2 \%$ $(45 / 68)$ of CEM had hypoechoic halos (22 cases with CDFI and 23 cases without), whereas $33.8 \%$ $(23 / 68)$ of nodules had no hypoechoic halos ( 11 cases with CDFI and 12 cases without). The finding demonstrated that the difference between the two groups was not statistically significant $(P=0.725$, $>0.05$ ) and that the blood flow signals and hypoechoic halos were not correlated.

The present study has limitations. First, there were several types of subcutaneous nodules at the superficial tissue yet we only compared CEM to EC but did not differentiate between CEM and other superficial soft tissue masses. Second, this being a single-center study with a limited number of selected patients, there may exist potential bias.

\section{Materials And Methods}

The demographic characteristics of the patients were reviewed from the outpatient/inpatient electronic medical records system. Ultrasonic imaging data were obtained from image workstations. Confirms that all experiments were performed in accordance with relevant named guidelines and regulations. The requirement for informed consent from patients was waived.

\section{Recruitment of subjects}

There were 309 cases enrolled in this study from December 2015 to December 2020. Inclusion criteria included: Patients whose lesions were surgically removed and the pathological results were CEM or EC; ultrasound was performed before the surgery in our hospital; complete clinical data were available. Exclusion criteria included: Patients who had not undergone surgery; the pathological results were not CEM or EC: incomplete medical records and imaging data. According to the time sequence of ultrasound examination, we categorized all subjects into the training set (between December 2015 and March 2019) and the validation set (between April 2019 and December 2020). Patients in the training set and the validation set were further divided into two groups, CEM and EC, according to the pathological results.

\section{Ultrasound evaluation}

All subjects underwent ultrasound evaluation, whereby we employed the LOGIQ E9 colour ultrasonic diagnostic apparatus (GE Healthcare, Wauwatosa, WI, USA) with a linear array probe (6-15 MHZ frequency). Ultrasound examination comprised grayscale and color Doppler flow imaging (CDFI). The subjects underwent a comprehensive scan of the lesions, including transverse, oblique, and longitudinal sections. The probe was placed gently over the lesion. The coupling agent was evenly coated on the 
lesion. Two sonographers with more than five years of experience interpreted the images of all subjects retrospectively; they were blinded from knowing the pathological results. The results of ultrasound evaluation were recorded, including size (length, width, thickness, length/thickness), location (head and face, neck, trunk, buttocks, limbs, axillary fossa, groin), morphology (round, oval, irregular), boundary (distinct, indistinct), echogenicity (hypoechoic, hyperechoic, isoechoic, anechoic, mixed), parenchyma echo(homogeneous, heterogeneous), posterior echo (enhanced, attenuated, invariant), calcification (without, dotted, patchy, full), a hypoechoic halo around nodular (with, without), special sign (fissure sign, apple sign, onionskin sign, dotted and striped hyperecho, without), CDFI (without, peripheral, internal, both of them). The two sonographers resolved any disagreement through consultation among themselves to reach a consensus before recording.

\section{Statistical analysis}

All statistical data were analysed using SPSS Statistics (IBM Corp. Released 2016; IBM SPSS Statistics for Windows, Version 24.0. Armonk, NY: IBM Corp.) and the software package R (version 3.5.3). The normal distribution of continuous variables was evaluated via the Kolmogorov-Smirnov test. Normally distributed data were expressed as the mean and standard deviation (), whereas data with a non-normal distribution were expressed by the median, 25th percentile (P25), and 75th percentile (P75). Categorical variables were presented as numbers and percentages. To evaluate the different factors between groups, independent samples t-test was executed on continuous variables with a normal distribution. MannWhitney U test was performed for nonconforming normal distributions. Pearson chi-square test or Fisher's exact test were applied for categorical variables. For multivariate analysis, logistic regression was employed. Variables with statistical significance in univariate analysis $(P<0.05)$ were included in the logistic regression model for multivariate analysis (Figure 1). Maximum Youden index values were applied to establish the optimal threshold cut-off values for prognostic factors as continuous variables based on the receiver operating characteristic (ROC). A diagnostic model was constructed as a nomogram. To assess the discriminatory capability of the diagnostic model, we applied the area under the curve (AUC) of the receiver operating characteristic (ROC) curve. $P$-values $<0.05$ was considered statistically significant.

\section{Declarations}

Acknowledgments: None.

Authors Contributions All authors have participated in the research ZFX and WXL designed the research, PKK, ZLL and LY collected and collated the cases data. SCC and XJ performed the literatures search and statistical analysis, NPJ and WM interpreted the data, ZYQ and CDF drafted the manuscript. ZFX is Corresponding Author. ZFX and WXL made critical revision of the manuscript. All authors read and approved the final manuscript. All authors agreed to be accountable for all aspects of the work in ensuring that questions related to the accuracy or integrity of any part of the work are appropriately investigated and resolved. 
Data Availability Statement: All data generated or analysed during this study are included in this published article.

Conflicts of interest: The author(s) declare no competing interests.

\section{References}

1. Roche, N. A., Monstrey, S. J. \& Matton, G. E. Pilomatricoma in children: common but often misdiagnosed. Acta Chir Belg 110, 250-254, doi:10.1080/00015458.2010.11680612 (2010).

2. Hsieh, T. J., Wang, C. K., Tsai, K. B. \& Chen, Y. W. Pilomatricoma: magnetic resonance imaging and pathological evaluation. J Comput Assist Tomogr 32, 320-323 (2008).

3. Lin, S.-F., Xu, S.-H. \& Xie, Z.-L. Calcifying epithelioma of malherbe (Pilomatrixoma): Clinical and sonographic features. Journal of Clinical Ultrasound 46, 3-7, doi:10.1002/jcu.22517 (2018).

4. Solivetti, F. M. et al. Epithelioma of Malherbe: new ultrasound patterns. J Exp Clin Cancer Res 29, 42, doi:10.1186/1756-9966-29-42 (2010).

5. Lee, D. H. et al. Ultrasound Feature-Based Diagnostic Model Focusing on the "Submarine Sign" for Epidermal Cysts among Superficial Soft Tissue Lesions. Korean J Radiol 20, 1409-1421, doi:10.3348/kjr.2019.0241 (2019).

6. Kim, H. K., Kim, S. M., Lee, S. H., Racadio, J. M. \& Shin, M. J. Subcutaneous epidermal inclusion cysts: ultrasound (US) and MR imaging findings. Skeletal Radiol 40, 1415-1419 (2011).

7. Chiu, Y. H., Chang, K. V., Chen, I. J., Wu, W. T. \& Özçakar, L. Utility of sonoelastography for the evaluation of rotator cuff tendon and pertinent disorders: a systematic review and meta-analysis. Eur Radiol 30, 6663-6672, doi:10.1007/s00330-020-07059-2 (2020).

8. Pressney, I., Khoo, M., Hargunani, R. \& Saifuddin, A. Description of the MRI and ultrasound imaging features of giant epidermal cysts. Br J Radiol 93, 20200413 (2020).

9. Herrmann, J. L., Allan, A., Trapp, K. M. \& Morgan, M. B. Pilomatrix carcinoma: 13 new cases and review of the literature with emphasis on predictors of metastasis. J Am Acad Dermatol 71, 38-43 e32, doi:10.1016/j.jaad.2014.02.042 (2014).

10. Liu, R. et al. MRI findings of an atypical testicular epidermoid cyst: A case report. Medicine (Baltimore) 99, e18818, doi:10.1097/MD.0000000000018818 (2020).

11. Liu, R., Lei, Z., Chen, N., Fang, J. \& Zhang, Y. Imaging in testicular epidermoid cysts. Clin Imaging 50, 211-215, doi:10.1016/j.clinimag.2018.03.020 (2018).

12. Pires-Gonçalves, L., Silva, C., Teixeira, M., Costa-Dias, S. \& Sousa-Mendes, V. Testicular epidermoid cyst - Ultrasound and MR typical findings with macroscopy correlation. Int Braz J Urol 37, 534-535, doi:10.1590/s1677-55382011000400014 (2011).

13. Kwon, D., Grekov, K., Krishnan, M. \& Dyleski, R. Characteristics of pilomatrixoma in children: a review of 137 patients. Int J Pediatr Otorhinolaryngol 78, 1337-1341, doi:10.1016/j.ijporl.2014.05.023 (2014). 
14. Cappellesso, R. et al. YAP immunoreactivity is directly related to pilomatrixoma size and proliferation rate. Arch Dermatol Res 307, 379-383, doi:10.1007/s00403-014-1530-2 (2015).

15. de Mendonça, J. C. G. et al. Epidermoid Cyst: Clinical and Surgical Case Report. Ann Maxillofac Surg 7, 151-154, doi:10.4103/ams.ams_68_16 (2017).

16. Schwarz, Y., Pitaro, J., Waissbluth, S. \& Daniel, S. J. Review of pediatric head and neck pilomatrixoma. Int J Pediatr Otorhinolaryngol 85, 148-153, doi:10.1016/j.ijporl.2016.03.026 (2016).

17. Ieni, A. et al. Limits of fine-needle aspiration cytology in diagnosing pilomatrixoma: a series of 25 cases with clinico-pathologic correlations. Indian J Dermatol 57, 152-155, doi:10.4103/00195154.94295 (2012).

18. Cecen, E. et al. Pilomatricoma in children: a frequently misdiagnosed superficial tumor. Pediatr Hematol Oncol 25, 522-527, doi:10.1080/08880010802235181 (2008).

19. Sivakumar, S. Pilomatrixoma as a diagnostic pitfall in fine needle aspiration cytology: a case report. Acta Cytol 51, 583-585 (2007).

20. Cevik, H. B., Erkan, M., Kayahan, S., Bulut, G. \& Gumustas, S. A. A skin tumor from an orthopedic oncology perspective: Pilomatrixoma in extremities (11 years experience with 108 cases). Dermatol Ther 32, e13004, doi:10.1111/dth.13004 (2019).

21. Thinakaran, V., Singh, S. K., Simples, P. \& Nadimpalli, V. Fine needle aspiration diagnosis of pilomatrixoma. A case report. Acta Cytol 42, 769-771 (1998).

22. Lee, H. S. et al. Relationship between sonographic and pathologic findings in epidermal inclusion cysts. J Clin Ultrasound 29, 374-383 (2001).

23. Solivetti, F. M. et al. Sonographic appearance of sebaceous cysts. Our experience and a review of the literature. Int J Dermatol 58, 1353-1359, doi:10.1111/ijd.14515 (2019).

24. Hwang, J. Y., Lee, S. W. \& Lee, S. M. The common ultrasonographic features of pilomatricoma. J Ultrasound Med 24, 1397-1402 (2005).

25. Lim, H. W. et al. Pilomatricomas in children: imaging characteristics with pathologic correlation. Pediatr Radiol 37, 549-555, doi:10.1007/s00247-007-0461-x (2007).

26. Nigam, J. S. et al. Epidermal Cysts: A Clinicopathological Analysis with Emphasis on Unusual Findings. Int J Trichology 9, 108-112, doi:10.4103/ijt.ijt_16_17 (2017).

27. Yuan, W. H., Hsu, H. C., Lai, Y. C., Chou, Y. H. \& Li, A. F. Differences in sonographic features of ruptured and unruptured epidermal cysts. J Ultrasound Med 31, 265-272 (2012).

28. Choo, H. J. et al. Pilomatricomas: the diagnostic value of ultrasound. Skeletal Radiol 39, 243-250, doi:10.1007/s00256-009-0678-x (2010).

29. DeRosa, D. C. \& Lin-Hurtubise, K. Pilomatricoma: an unusual dermatologic neoplasm. Hawaii J Med Public Health 71, 282-286 (2012).

\section{Figures}




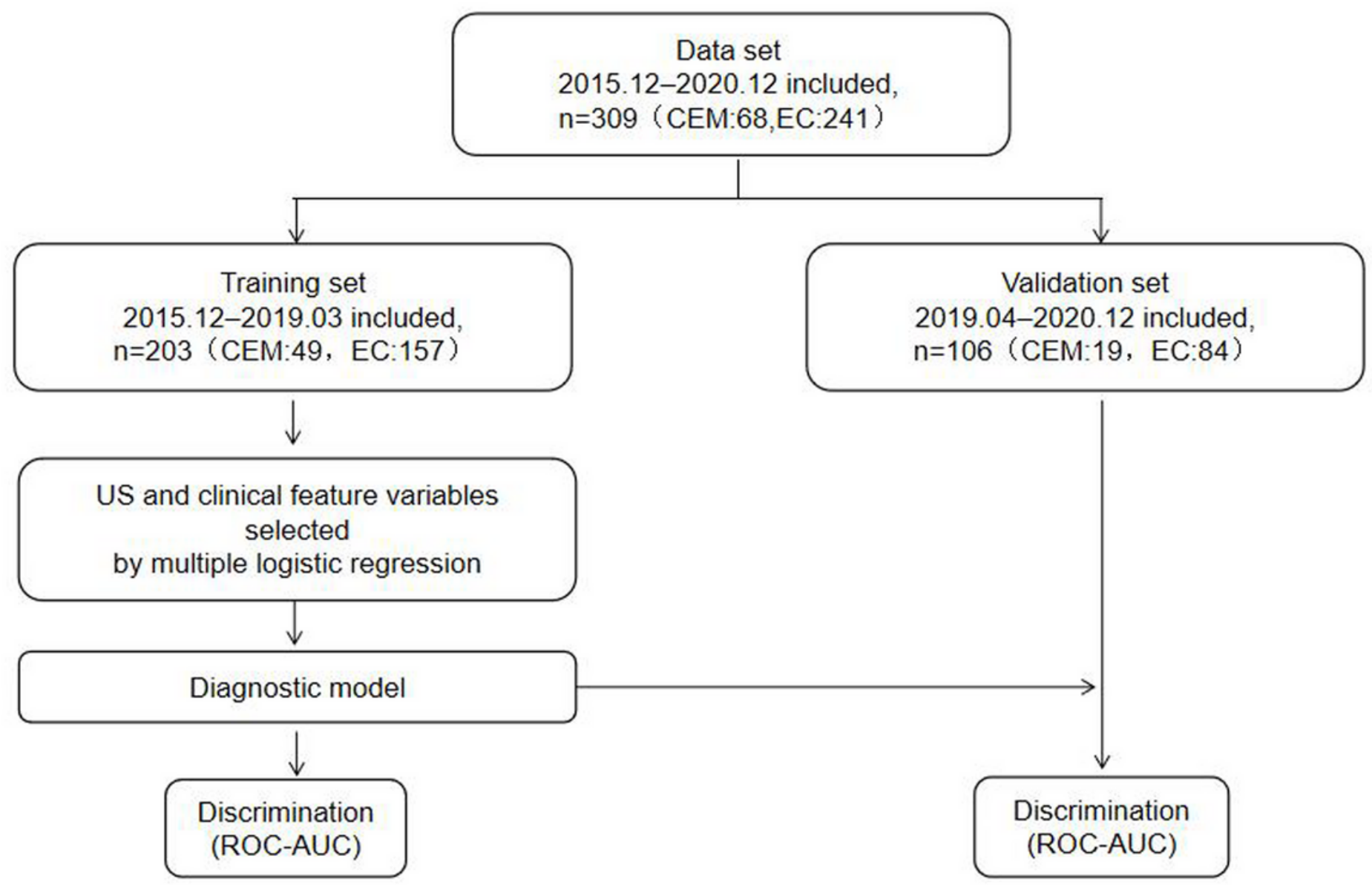

Figure 1

Statistical analysis scheme. 
Points

\begin{tabular}{|c|c|c|c|c|c|c|c|c|c|}
\hline 10 & 20 & 30 & 40 & 50 & 60 & 70 & 80 & 90 & 100 \\
\hline
\end{tabular}

age

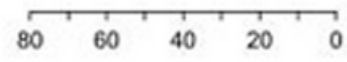

thickness

\begin{tabular}{llllllllll}
\hline 18 & 16 & 14 & 12 & 10 & 8 & 6 & 4 & 2 & 0
\end{tabular}

posterior echo

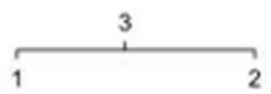

hypoechoic halo

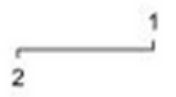

Total Points

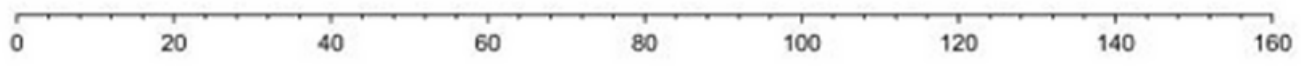

Predicted Value

$0 \longdiv { 0 . 3 0 . 5 0 . 7 } 0 . 9$

\section{Figure 2}

The nomogram for discriminating CEM nodules.

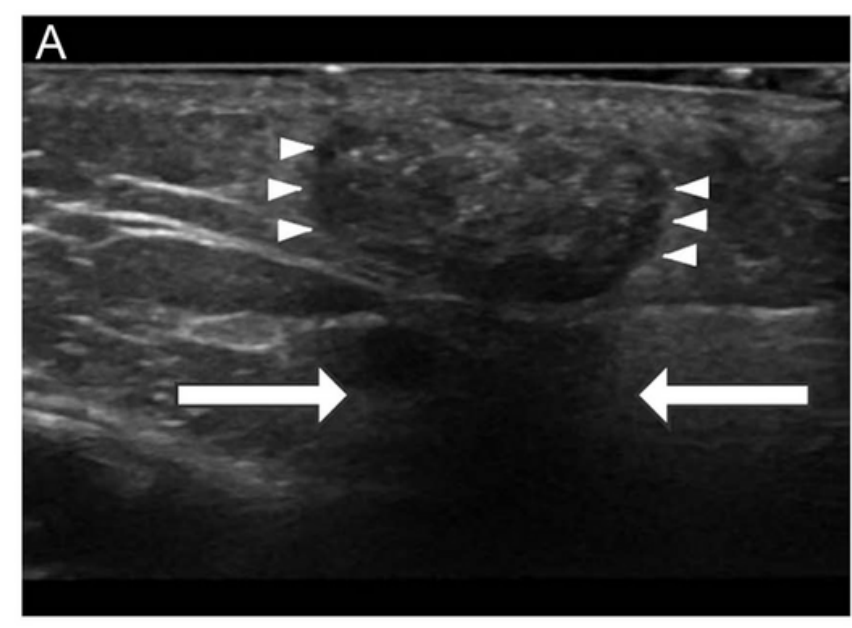

B Points

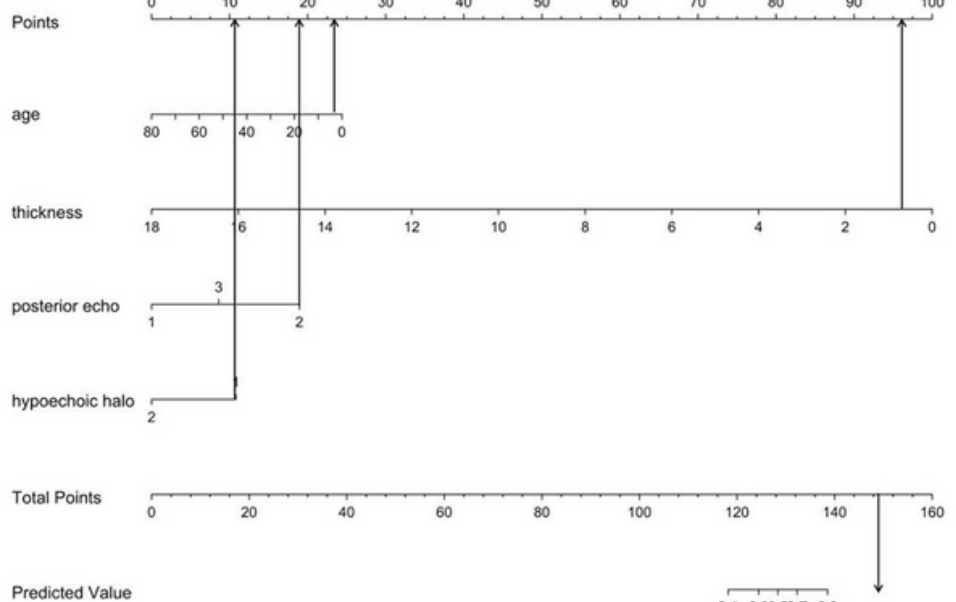

Predicted Value

0.10 .30 .50 .70 .9

\section{Figure 3}

The case of CEM identified using the constructed nomogram. 
(3A) The patient was 1 year-old and had hypoechoic nodules on the subcutaneous tissue of the right face with hypoechoic halos (arrowheads), posterior echo attenuation (arrows) via ultrasound. The thickness of the nodules was $0.7 \mathrm{~cm}$. (3B) Total point which was taken from nomogram of patient was $151(23+96+$ $19+11=149$ ). This point was converted to a predicted probability of $>0.90$. A similar numerical value was obtained by visual assessment of the nomogram (arrow). The pathologic result was CEM.
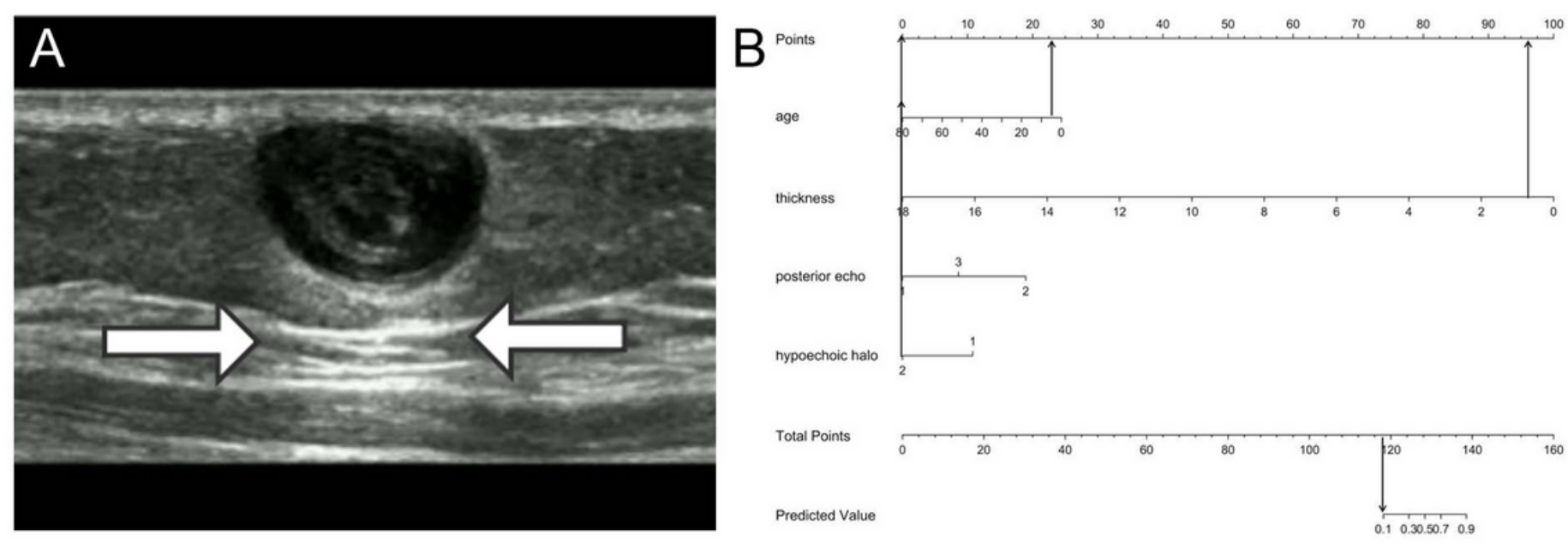

\section{Figure 4}

The case of EC identified using the constructed nomogram.

(4A) The patient was 9 year-old and had hypoechoic nodules on the subcutaneous tissue of the right abdominal wall with posterior echo enhancement (arrows) and without hypoechoic halos via ultrasound. The thickness of the nodules was $0.7 \mathrm{~cm}$.

(4B) Total point which was taken from nomogram of patient was $117(22+96+0+0=118)$. This point was converted to a predicted probability of 0.1 . A similar numerical value was obtained by visual assessment of the nomogram (arrow). The pathologic result was EC. 


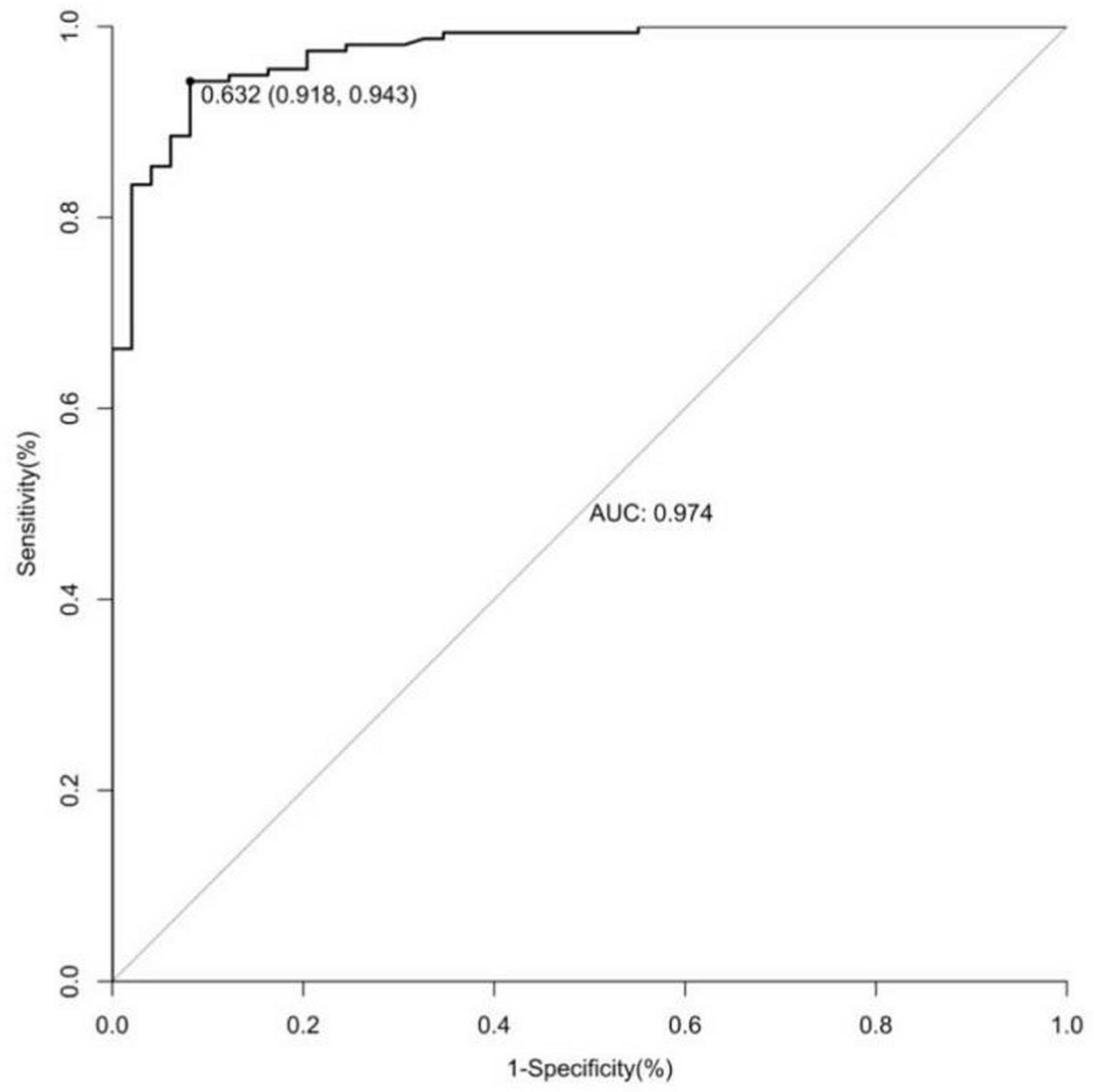

Figure 5

ROC curve of the training set.

AUC was 0.974 (95\%Cl: 0.955-0.994). 


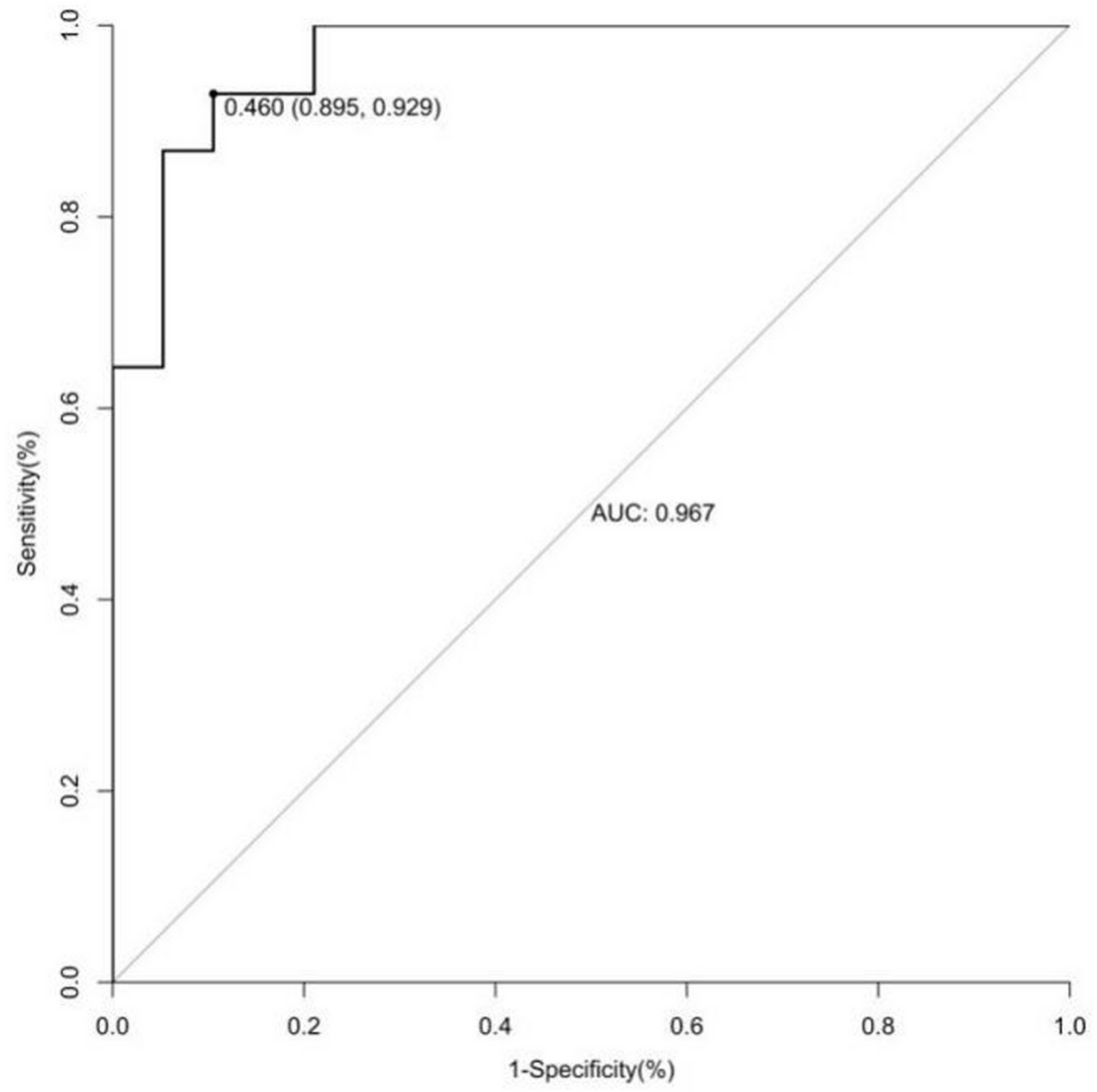

Figure 6

ROC curve of the validation set.

AUC was 0.967 (95\%Cl: 0.926-1.000). 